\title{
ON THE SPAN OF A RIEMANN SURFACE
}

\author{
BY BURTON RODIN
}

Communicated by Fred Gehring, October 31, 1969

In 1952 Virtanen [7] gave an example of a Riemann surface which carries nonconstant $A D$-functions, yet there is a point on the surface where every such function has a critical value, i.e. a vanishing derivative. It had already been pointed out in [1] that this phenomenon cannot occur for planar surfaces. It has remained an open question whether this situation prevails for harmonic functions. This unsolved problem was restated in the monograph Capacity functions (SarioOikawa [5]). Problem 4 in the list of open questions at the end of that book asks, more generally, whether the vanishing of the span $S_{m}$ at one point on a Riemann surface implies that it vanishes at all points on the surface.

In this paper we describe a Riemann surface $R$ with the property that for any preassigned positive integer $m$ there is a point on $R$ where every $H D$-function has a critical point of order $m$. Yet $R$ carries nonconstant $H D$-functions. Thus Problem 4 of [5] is solved for the $H$-span. Nevertheless, there are still unresolved questions in this area when we restrict ourselves to surfaces of finite genus or to $K D$ functions.

Consider the surface of Tôki [6] (this surface is also constructed in Ahlfors-Sario [2]; see No. 25 of $\S 8$, Chapter IV). This surface is realized as a unit disk with infinitely many radial slits identified in a prescribed manner. It is of class $O_{H D}-O_{G}$, and hence the Royden harmonic boundary [4] consists of exactly one point.

Remove a disk $\{|z|<\epsilon\}$ from Tôki's surface and then form the double across $\{|z|=\epsilon\}$. The resulting surface is realized as a radial slit annulus $\left\{\epsilon^{2}<|z|<1\right\}$ with certain identifications among the edges of the slits. For a preassigned positive integer $m$, insert an additional $m+1$ radial slits symmetric to the origin and identify their edges cyclically. The resulting surface $R$ has a branch point of order $m$, and the Royden harmonic boundary of $R$ consists of two points. Therefore the space of $H D$-functions on $R$ is two-dimensional.

The function $\log r$ can be regarded as an $H D$-function on $R$, and in that sense it has critical point of order $m$ at the branch point of order $m$. The same is true for any $H D$-function $u$ on $R$ since

AMS Subject Classifications. Primary 3045; Secondary 3111.

Key Words and Phrases. Harmonic functions, Dirichlet finite harmonic functions, $H D$-functions, critical points, span, Royden harmonic boundary, capacity functions. 


$$
u=c_{1} \log r+c_{2} \text {. }
$$

We see that $R \notin O_{H D}$, yet there is a point on $R$ where the $m$-span $S_{m}$ vanishes.

\section{REFERENCES}

1. L. V. Ahlfors and A. Beurling, Conformal invariants and function-theoretic nullsets, Acta Math. 83 (1950), 101-129. MR 12, 171.

2. L. V. Ahlfors and L. Sario, Riemann surfaces, Princeton Math. Series, no. 26, Princeton Univ. Press, Princeton, N. J., 1960. MR 22 \#5729.

3. L. Sario, Über Riemannsche Flächen mit hebbarem Rand, Ann. Acad. Sci. Fenn. Ser. A. I. Math.-Phys. No. 50 (1948). MR 10, 365.

4. H. L. Royden, On the ideal boundary of a Riemann surface. Contributions to the theory of Riemann surfaces, Ann. of Math. Studies, no. 30, Princeton Univ. Press, Princeton, N. J., 1953. MR 15, 25.

5. L. Sario and K. Oikawa, Capacity functions, Springer-Verlag, Berlin and New York, 1969.

6. Y. Tôki, On the classification of open Riemann surfaces, Osaka Math. J. 4 (1952), 191-201. MR 14, 864.

7. K. I. Virtanen, Über Extramalfunktionen auf offenen Riemannschen Flächen, Ann. Acad. Sci. Fenn. Ser. A. I. Math.-Phys. No. 141 (1952). MR 14, 743.

University of California at San Diego, la Jolla, California 92038 